\title{
Which proliferation markers for routine immunohistology? A comparison of five antibodies
}

\author{
D S C Rose, P H Maddox, D C Brown
}

\begin{abstract}
Aims-To determine the best of five antibodies for immunohistochemical assessment of growth fraction in formalin fixed, paraffin wax embedded tissues.

Methods-Sections from 100 recent, and 17 ten year old or over wax embedded blocks of normal and malignant tissues were immunostained with monoclonal Ki67, polyclonal Ki67, PC10, MIB1, and JC1. The antibodies were evaluated for specificity of nuclear versus cytoplasmic staining, cleanliness of background, and compared with the expected pattern of staining in normal tissues, defined immunohistochemically by monoclonal Ki67 antibody in frozen tissues or by tritiated thymidine uptake.

Results-No marker was ideal, but best results were obtained with MIB1 and polyclonal Ki67, followed by JC1, PC10, and monoclonal Ki67.

Conclusions-For routine use, MIB1 or polyclonal Ki67 are the best proliferation markers in conventional histological preparations. The other markers tested cannot be recommended.
\end{abstract}

(F Clin Pathol 1994;47:1010-1014)

Measurement of cell proliferation may provide useful information concerning tumour prognosis and aid diagnosis, especially for low grade non-Hodgkin's lymphomas. ${ }^{1-6}$ Methods directed at different parts of the cell cycle are available. Each has advantages and disadvantages. ${ }^{6}$ Mitotic counts ( $M$ phase) give diagnostic and prognostic information for some tumours, such as smooth muscle tumours, and are used as an adjunct to tumour grading-for example, in breast carcinoma. ${ }^{8}$ Mitoses counting is simple to perform, but errors can occur ${ }^{9}$ because of delays in fixation, ${ }^{10}$ section thickness, the size of the microscope field, ${ }^{11}$ and difficulties in the recognition of mitoses. DNA synthesis ( $\mathrm{S}$ phase) can be assayed by measuring labelled DNA precursor incorporation, such as tritiated thymidine, or a nucleotide analogue, such as bromodeoxyuridine (BrDU). These methods permit direct estimation of DNA synthesis and are therefore the standards against which other methods must be assessed. Tritiated thymidine in tissue sections can be revealed with photographic emulsion, and $\mathrm{BrDU}$ can be revealed immunohistochemically, enabling the morphology of proliferating cells to be identified. Alternatively, scintography or flow cytometry can give an objective measure of large numbers of cells labelled with these reagents. However, both methods require in vivo administration or in vitro ${ }^{12}$ incubation of tissue with associated ethical and logistic problems, preventing widespread clinical use. Many routine laboratories will also not want to use radionucleotides. Flow cytometry can provide a quantitative measurement of the DNA content of large numbers of cells from fresh, fixed, or paraffin wax embedded tissue. ${ }^{13}$ All nucleated cells contain DNA throughout the cell cycle, so flow cytometry measures ploidy rather than proliferation fraction. Statistical correction for distortions due to aneuploidy must be used to isolate the $S$ phase of tumours. ${ }^{14}$ Flow cytometry requires dissociation of nuclei, permitting contamination of tumour samples by reactive cells and preventing morphological analysis. Silver staining of proteins associated with nucleolar organiser regions (AgNORs) of interphase chromosomes may be used on conventional histological sections for assessment of proliferation rates; correlation with other indices of cellular proliferation has been shown. ${ }^{15}$ The value of AgNOR counting to discriminate between benign and malignant lesions, and the relevance of AgNOR counts to prognosis is not clear. ${ }^{16} 17$

Several antibodies to cell cycle related antigens are available. Monoclonal Ki67 is the prototypic antibody, ${ }^{18}$ recognising a nuclear protein $^{19}$ expressed in proliferating cells in G1, S, G2, and $M$ but not G0. ${ }^{20} \mathrm{Ki67}$ labelling correlates well with cell proliferation as assessed by tritiated thymidine uptake, ${ }^{20}$ and with histological grade and prognosis in non-Hodgkin's lymphomas. ${ }^{2}$ A major limitation of the use of monoclonal $\mathrm{Ki} 67$ is the requirement for fresh or frozen tissue because fixation abolishes staining in most but not all laboratories. ${ }^{21}$ Newer antibodies, polyclonal Ki67 and MIB1, raised against peptides from recombinant fragments of the gene for $\mathrm{Ki} 67$ antigen, are effective in conventional sections following microwave irradiation. ${ }^{22}{ }^{23} \mathrm{JC} 1$ is a monoclonal antibody which has been reported to identify a nuclear protein associated with cell proliferation. ${ }^{24}$ Proliferating cell nuclear antigen (PCNA) is a nuclear protein associated with DNA polymerase $\delta^{25}{ }^{26}$ which is present throughout the cell cycle in proliferating cells. The monoclonal antibody PC10 recognises PCNA and is effective in formalin fixed, paraffin wax embedded tissues. ${ }^{27}$ It is therefore a potentially useful proliferation marker, 
but has been shown to stain non-proliferating cells in some circumstances. ${ }^{27}{ }^{28} \mathrm{PC} 10$ is sensitive to the length of tissue fixation before processing $^{28}$ and section handling. ${ }^{29}$

Because most diagnostic laboratories use immunohistochemistry, we evaluated five immunohistochemical markers of proliferation (three to Ki67, one to PCNA, and one to JC1), specifically from the viewpoint of a diagnostic pathologist. Our aim was to find the marker that best matched the proliferation profile of normal and abnormal tissues as defined by established methods.

\section{Methods}

Paraffin wax blocks of recently processed formalin fixed normal tissues and tumours were retrieved from the files of the Histopathology Department, Whittington Hospital, together with archival tissue processed at least 10 years previously. The tissue had been fixed in neutral buffered $10 \%$ formol-saline and fixation periods ranged from 12 hours to several days. Tissues stained included one archival and five recent samples of each of the following: normal stomach, small intestine, colon, liver, skin, reactive lymph node, kidney, and testis, five (recent samples only) of thyroid and fetal thymus; one archival and five recent samples of each of the following: cutaneous basal cell carcinoma, carcinoma of stomach, colon, breast, endometrium, kidney, prostate, bladder; and five (recent samples only) of squamous cell carcinoma of the cervix. One archival and five recent leiomyosarcomas of the uterus were also stained. For each carcinoma category, a similar grade of tumour was used.

To assess large numbers of samples under very similar conditions, multiblocks were prepared as described before. ${ }^{30}$ Use of the multiblock technique for individual tumour prognosis has been criticised on the grounds of sampling error ${ }^{31}$ but these objections do not apply to our study. Multiblocks are ideal for the comparison of different antibodies because sequential sections are stained and intertumoural comparisons of proliferation rates were not attempted. The primary and secondary antibodies used in this study are presented in table 1.

In accordance with standard practice and the manufacturer's suggestions, we determined the optimum antibody concentrations before performing the study.

Preliminary experiments with the antibodies on untreated sections obtained moderate results with $\mathrm{PC} 10$ and poor results with the

Table 1 First and second layer antibodies

\begin{tabular}{llll}
\hline Antibody & $\begin{array}{l}\text { Suggested } \\
\text { concentration }\end{array}$ & $\begin{array}{l}\text { Working } \\
\text { concentration }\end{array}$ & $\begin{array}{l}\text { Second } \\
\text { layer }\end{array}$ \\
\hline Monoclonal Ki67 & Neat & Neat & RAM \\
Polyclonal Ki67 (Dako; A047) & 1 in 90 1 in 50 to 1 in 100 & 1 in 90 & SAR \\
PC10 (Dako; M879) & 1 in 100 & RAM \\
MIB1 (Immunotech; 0505) & 1 in 50 to 1 in 100 & 1 in 100 & RAM \\
JC1 & Neat & Neat & RAM \\
\hline
\end{tabular}

RAM = biotinylated rabbit anti-mouse; SAR = biotinylated swine anti-rabbit. others. Trypsin abolished staining with all five antibodies. Therefore, we used microwave postfixation as many antibodies show enhanced staining after microwave irradiation of conventionally processed sections. ${ }^{32}$

Sections $(4 \mu \mathrm{m})$ were cut on to organosilane coated ${ }^{33}$ slides and dried at $38^{\circ} \mathrm{C}$ to prevent loss of PCNA that can occur with drying at $60^{\circ} \mathrm{C}^{29}$ Slides were dewaxed and endogenous peroxidase blocked with hydrogen peroxide in methanol. Microwave postfixation was performed using a domestic oven (Kenwood KM 2001T) at $700 \mathrm{~W}$, delivered to slides immersed in $0.01 \mathrm{M}$ citrate buffer, pH 6.0 , as two five minute doses separated by a two minute break, enabling refill of buffer if required. The slides were permitted to cool to room temperature before removal. Sections were incubated with primary layer antibodies at $4^{\circ} \mathrm{C}$ overnight. Biotinylated rabbit or swine second layer antibodies (table 1 ) were applied for 60 minutes at room temperature. Bound antibody was visualised with avidin-peroxidase conjugate (Dako K355, High Wycombe, UK) and diaminobenzidine (Sigma D5905, St Louis, Missouri, USA). Sections were lightly counterstained with Mayer's haematoxylin. Negative control sections were included. The slides were labelled with a code so that an independent assessment by two pathologists would be unprejudiced. Semiquantative scoring was used as in previous studies of proliferation antigens. ${ }^{328}$

Sections were scored for:

(1) Intracellular distribution of antibody, scoring 1 for restriction to the nucleus, 0 for the presence of cytoplasmic positivity. Cells in mitosis and the basal layers of the epidermis were not included because cytoplasmic staining is expected in mitotic cells and in the basal layers of the epidermis when frozen tissue is stained by monoclonal Ki67. ${ }^{18} 24$

(2) Degree of background staining, scoring 2 for clean nuclear staining, 1 for cytoplasmic staining not preventing interpretation of proliferation, 0 for uninterpretable.

(3) Expected pattern of staining was scored with reference to the distribution of proliferating cells determined by monoclonal Ki67 in frozen tissue or tritiated thymidine uptake ${ }^{18}$; antibodies scoring 3 for faithful reproduction of pattern, 2 for excess positive cells, 1 if most cells stained.

With the multiblock technique, if one tissue sample is lost from a multiblock section it is often lost throughout sequential sections. We excluded three of 117 tissue sample sections from the analysis because of section loss.

\section{Results}

The major difference between antibodies was in degree of cytoplasmic staining, the cleanliness of the background, and in the restriction of specificity (table 2 ).

It is not immediately apparent why there was a discrepancy between the degree of nuclear versus cytoplasmic staining and the cleanliness of background staining. When scoring distribution of the stain, however, 
Table 2 Comparison of staining performance by each antibody expressed as percentage of maximum (100\%) possible score

\begin{tabular}{|c|c|c|c|c|c|}
\hline \multirow[b]{2}{*}{ Index } & \multicolumn{5}{|l|}{ Antibody } \\
\hline & $\begin{array}{l}\text { Monoclonal } \\
\text { Ki67 }\end{array}$ & $\begin{array}{l}\text { Polyclonal } \\
\text { Ki67 }\end{array}$ & PC10 & $M I B 1$ & FC1 \\
\hline $\begin{array}{l}\text { Nuclear } v \text { cytoplasmic } \\
\text { staining }{ }^{\star} \\
\text { Background }^{\star} \\
\text { Expected pattern }^{\star \star}\end{array}$ & $\begin{array}{r}6.5 \\
13 \cdot 2 \\
20.2\end{array}$ & $\begin{array}{l}92.6 \\
93.0 \\
88.5\end{array}$ & $\begin{array}{l}38.9 \\
70 \cdot 0 \\
38.5\end{array}$ & $\begin{array}{l}93.5 \\
93.8 \\
95 \cdot 2\end{array}$ & $\begin{array}{l}62 \cdot 0 \\
75 \cdot 8 \\
56 \cdot 7\end{array}$ \\
\hline
\end{tabular}

* Scoring for 114 of 117 tissue sample sections (three lost)

$\star \star$ Expected pattern for 52 of 53 tissue sample sections of non-neoplastic tissue for which data on expected distribution were known (one lost).

cytoplasmic positivity gained no points; in scoring background, if the nuclei were rendered unassessable the score was 0 , but if the cytoplasmic staining did not prevent interpretation, one point was given. The best antibodies gave sufficiently little cytoplasmic staining that the discrepancy between the two scoring methods was negligible.

Monoclonal Ki67 stained nuclei and cytoplasm in almost all cells in the sections, often preventing any meaningful assessment of proliferation status.

PC10 and JC1 were more specific with less cytoplasmic staining but the antibodies still stained most cell nuclei. However, intensity of staining varied such that-for example, nuclei in follicle centres in lymph nodes stained more strongly than in the mantle zones, but differences between follicle centre cells could not be visualised. Polyclonal Ki67 and MIB1 compared well with proliferation patterns in normal tissues, as defined by DNA precursor uptake studies or immunohistochemistry using monoclonal $\mathrm{Ki} 67$ in frozen tissue. ${ }^{18}$ Both were largely confined to nuclei, with the most common pattern being a granular nuclear stain with highlighting of nucleoli and, occasionally, the nuclear membrane. Both Ki67 and MIB1 stained renal tubular cytoplasm, and one renal cell carcinoma with a very granular, proximal convoluted tubulelike appearance. Staining of tubular cytoplasm was largely responsible for the less than $100 \%$ score obtained by these antibodies on assessment of background. This non-specific staining of renal tubules is not restricted to proliferation markers (personal observations, Rose DSC). Of the well differentiated tumours, the basal cell carcinomas and papillary transitional cell carcinomas often showed basal layer staining, but no staining of the more mature upper epithelial layers. In dividing cells mitotic figures stained strongly, with weak diffuse cytoplasmic positivity. Polyclonal Ki67 scored worse than MIB1 for adherence to the expected pattern because of lack of staining of early testicular germ cells. If the

Table 3 Comparison of summed results (all antibodies, all tissues) from archival (>10 years old) and recent samples expressed as percentage of maximum (100\%) possible score

\begin{tabular}{lll}
\hline Index & Archival & Recent \\
\hline Nuclear v cytoplasmic staining & 63.8 & 57.8 \\
Background & 70.7 & 68.2 \\
Expected & 57.5 & 60.6 \\
\hline
\end{tabular}

sections of testicular tissue are excluded from the analysis, the expected pattern score for polyclonal $\mathrm{Ki} 67$ is $93.5 \%$, very similar to MIB1.

If normal testis is to be examined MIB1 is preferable. In all tissues examined MIB1 gave a more intense positive stain, affording greater positive versus negative contrast and a more confident categorisation of nuclei, often at lower magnification. The effect is difficult to quantify, and so on objective analysis according to the parameters we measured, the two antibodies must rank equally.

We found no difference in the staining performance of the antibodies in archival as opposed to recent tissues (table 3).

\section{Discussion}

Over the past 10 years, there has been an explosion of interest in the measurement of the proliferation fraction of tumours and normal tissues. There are two practical applications of interest to a diagnostic pathologist.

The first relates to tumour prognosis. Staging and grading systems are commonly used in reporting carcinomas of breast, colon, bladder, prostate, and melanomas. Biological markers of prognosis are not often used, largely because of the requirement for fresh tissue. Hormone receptor status in breast carcinoma is the exception ${ }^{34}$ and now a reliable immunohistochemical marker for formalin fixed tissue is available. ${ }^{35}$

The proliferation fraction, whatever the measurement method, has been correlated with prognosis for many tumours, ${ }^{1}$ especially breast $^{45}$ and lymphoid neoplasms. ${ }^{2}$ However, several authors have urged caution because different methods measure different indices, not all directly related to the proliferation fraction. ${ }^{636}$ In this study we used three antibodies directed against the $\mathrm{Ki} 67$ antigen, one to the JC1 antigen, and one to PCNA. The latter is expressed continuously in proliferating cells and shows good correlation with $\mathrm{BrDU}$ and $\mathrm{Ki} 67$ determined proliferation in normal cells and non-Hodgkin's lymphomas. ${ }^{27}$ In cultured HeLa cells, however, the correlation between the PCNA measured proliferation fraction and the $S$ phase fraction as assessed by flow cytometry is poor. ${ }^{27}$ In rapidly growing tumours this may be because of the long $\mathrm{t}_{1 / 2}$ of $\mathrm{PCNA}^{37}$ relative to the rate of cell turnover. PCNA staining may also be seen in non-proliferating (Ki67 negative) hepatocytes adjacent to tumours, and in hepatitis, ${ }^{28}$ possibly because of the enhancement of PCNA expression by growth factors released from the tumour cells or the inflammatory infiltrate. Similar excess staining is seen in biopsy specimens of normal and inflamed gastric mucosa. ${ }^{38}$ PC10 staining in these circumstances may not indicate proliferation even though it may be technically faultless in showing the presence of PCNA. Conversely, $\mathrm{Ki} 67$ expression is regarded as a good index of the proliferation fraction as long as the antibody and staining method have been validated. 
The second practical application concerns diagnosis. New techniques are often welcomed in the hope that they will contribute to decision making in differential diagnosis. Deficiencies often emerge, particularly in the grey areas between reactive hyperplasia, dysplasia, and malignancy. Proliferation markers are no different. However, preliminary investigations have shown the value of staining for Ki67 antigen in one difficult differential diagnosis: reactive hyperplasia of lymph nodes versus follicular lymphoma. MIB1 staining of reactive lymph nodes reveals a high proliferation fraction in the follicle centres with prominent zonation. Centroblasts in the dark zone stain more strongly than centrocytes in the light zone. Proliferation centres in follicular lymphoma lack zonation and stain much less strongly than in reactive follicle centres. In combination with immunostaining for the product of the antiaptosis gene, $b c l-2$, this is a useful technique for the distinction between the two conditions (personal communication, Isaacson PG).

One possible criticism of our study is that the target tissues did not have a uniform fixation period. We deliberately selected a wide range of normal and abnormal tissues from archival samples to reflect routine diagnostic practice in which specimens are not usually received fresh and are inevitably exposed to variable rates and duration of fixation. Many of the larger specimens had at least 36 hours of fixation and tissues from a Friday evening operation may not enter the processor cycle until 80 hours later. This may cause problems with some antibodies. PCNA, as assessed by PC10, is very fixation dependent. Positivity in proliferating cells is reduced after 48 hours fixation and is almost abolished at 72 hours. ${ }^{27}$

Antigen retrieval from fixed material using protease treatment is an established method. Recently, there has been increasing interest in the use of microwave heating as an alternative. It often gives equivalent or superior results. ${ }^{39}$ Protease digestion has to be carefully controlled to prevent artefactual changes in antigen exposure and similar problems could be anticipated for microwave treatment. In an analysis of 256 antibodies, including PC10 and MIB1, Cattoretti et $a l^{32}$ found no adverse effects on staining specificity following microwave irradiation. However, divergence from the normal pattern of antibody binding can be seen as in a study of microwaved sections of lymph nodes from cases of Hodgkin's disease stained with CD 15 and CD $30 .{ }^{40} \mathrm{We}$ followed a commonly used protocol, ${ }^{3241}$ placing the tissue in the microwave oven for two closely separated five minute exposures, partially because it was effective, and partially to impose a simple pretreatment protocol. Other investigators, using multiples of seven minute exposures, have shown that MIB1 staining of overfixed material is enhanced if the duration of exposure is increased from seven to 14 minutes. ${ }^{42}$ Surprisingly, even prolonged irradiation does not have an adverse effect on staining $^{42}$ so if overfixed tissues are to be examined, prolonged irradiation may be pre- ferred. The main requirement for good antigen retrieval is application of wet heat, which can be provided satisfactorily by a range of methods. 3239

The differences in performance of the antibodies tested is not surprising. The three antibodies to $\mathrm{Ki} 67$ were raised against different epitopes which probably vary in accessibility and resistance to denaturation. PC10 will underperform in a study (or in diagnostic practice) using overfixed tissues; this problem, in conjunction with deregulated expression, makes PC10 a poor choice for routine use.

In conclusion, reliable antibodies for the measurement of the proliferation fraction in current or old conventionally processed tissues are now available. The most suitable for routine work is either MIB1 or polyclonal Ki67.

We thank Dr D Y Mason for the gift of monoclonal Ki67 and JC1, Dako UK for generously supplying the microwave oven polyclonal Ki67 and PC10, and The Binding Site Ltd, Birmingham, UK, for donating MIB1.

1 Brown DC, Gatter KC. Monoclonal antibody Ki67: its use in histopathology. Histopathology 1990:17:489-503. use in histopathology. Histopathology 1990;17:489-503.
Hall PA, Richards MA, Gregory WM, d'Ardenne AJ Lister TA, Stansfeld AG. The prognostic value of $\mathrm{Ki67}$ immunostaining in non-Hodgkin's lymphoma. $\mathcal{f}$ Pathol 1988;154:223-35.

3 Gatter KC, Dunnill MS, Gerdes J, Stein H, Mason DY. New approach to assessing lung tumours in man. $\mathcal{f}$ Clin Pathol 1986;39:590-3.

4 Bouzubar N, Walker KJ, Griffiths K, Ellis IO, Elston CW, Robertson JFR, et al. Ki-67 immunostaining in primary breast cancer: Pathological and clinical correlations. $\mathrm{BrF}$ Cancer 1989;59:943-7.

5 Gentili C, Sanfilippo O, Sivestrini R. Cell proliferation and its relationship to clinical features and relapse in breast cancers. Cancer 1981;48:974-9.

6 Hall PA, Levison DA. Review: Assessment of cell proliferation in histological material. F Clin Pathol 1990;43 184-92.

7 Kempson RL, Bari W. Uterine sarcomas. Classification diagnosis, and prognosis. Hum Pathol 1970;1:331-49.

8 Elston CW. Grading of invasive carcinoma of the breast. In: Page DL, Anderson TJ, eds. Diagnostic histopatholog of the breast. Edinburgh: Churchill Livingstone, 1987 300-11.

9 Silverberg SG. Reproducibility of the mitosis count in the histologic diagnosis of smooth muscle tumours of the uterus. Hum Pathol 1976;7:451-4.

10 Cross SS, Start RD, Smith JHF. Does delay in fixation affect the number of mitotic figures in processed tissue? affect the number of mitotic

11 Ellis PSJ, Whitehead R. Mitosis counting-a need for reappraisal. Hum Pathol 1982;12:3-4.

12 Malaise EP, Chavandra N, Tubiana $\dot{M}$. The relationship between growth rate, labelling index and histological type of human solid tumours. Eur $\mathcal{f}$ Cancer 1973 9:305-12.

13 Quirke P, Dyson JED. Flow cytometry: Methodology and applications in pathology. F Pathol 1986;149:79-87.

14 Scott N, Cross D, Plumb MI, Dixon MF, Quirke P. An investigation of different methods of cell cycle analysis by flow cytometry in rectal cancer. $\mathrm{Br} f$ Cancer by flow cytom

15 Hall PA, Crocker J, Watts A, Stansfeld AG. A comparison of nucleolar organizer region staining and Ki-67 immunostaining in non-

16 Egan MJ, Crocker J. Nucleolar organiser regions in pathology. Brf Cancer 1992;65:1-7.

17 Darnton SJ, Gray MR, Crocker J. Another cautionary not on the use of PCNA and AgNORs as markers of proliferation. $\mathcal{f}$ Pathol 1992;168:87.

18 Gerdes J, Schwab U, Lemke H, Stein H. Production of a mouse monoclonal antibody reactive with a human nuclear antigen associated with cell proliferation. Int $f$ Cancer 1983;31:13-20.

19 Gerdes J, Li L, Schlueter C, Duchrow M, Wholenberg C, Gerlach C, et al. Immunobiochemical and molecular biologic characterization of the cell proliferation-associated nuclear antigen that is defined by monoclonal anti-
body Ki-67. Am $\mathcal{F}$ Pathol $1991 ; 138: 867-73$.

20 Gerdes J, Lemke H, Baisch H, Wacker HH, Schwab U, Gerdes J, Lemke H, Baisch H, Wacker HH, Schwab U,
Stein H. Cell cycle analysis of a cell proliferation-associStein $\mathrm{H}$. Cell cycle analysis of a cell proliferation-associ-
ated human nuclear antigen defined by the monoclonal ated human nuclear antigen defined by the mon

21 Cuevas E, Jones DB, Wright DH. Immunohistochemical 
detection of tumour growth fraction (Ki-67 antigen) in formalin-fixed and routinely processed tissues. F Pathol 1993;169:477-8.

22 Key G, Petersen JL, Becker MHG, Duchrow M, Schlüter $\mathrm{C}$, Askaa J, et al. New antiserum against $\mathrm{Ki}-67$ antigen suitable for double immunostaining of paraffin wax sections. F Clin Pathol 1993;46:1080-4.

23 Cattoretti G, Becker MHG, Key G, Duchrow C, Schlüter $\mathrm{C}, \mathrm{Galle} \mathrm{J}$, et al. Monoclonal antibodies against recombinant parts of the $\mathrm{Ki}-67$ antigen (MIB1 and MIB3) detect proliferating cells in microwave-processed formalin-fixed paraffin sections. F Pathol 1992;168:357-63.

24 Garrido MC, Cordell JL, Becker MHG, Key G, Gerdes J Jones $\mathrm{M}$, et al. Monoclonal antibody JC1: New reagen for studying cell proliferation. F Clin Pathol 1992;45: $860-5$

25 Bravo R, Frank R, Blundell PA, Macdonald Bravo $\mathrm{H}$ Cyclin/PCNA is the auxillary protein of DNA polymerase- $\delta$. Nature 1987;326:515-7.

26 Prelich G, Tan C-K, Kostura M, Mathews MB, So AG Downey KM, et al. Functional identity of proliferatin cell nuclear antigen and a DNA polymerase- $\delta$ auxillary protein. Nature 1987;326:517-20.

27 Hall PA, Levison DA, Woods AL, Yu CC-W, Kellock DB, Watkins JA, et al. Proliferating cell nuclear antigen (PCNA) immunolocalisation in paraffin sections: an index of cell proliferation with evidence of deregulated index of cell proliferation with evidence of deregulated expression.

28 Harrison RF, Reynolds GM, Rowlands DC. Immunohistochemical evidence for the expression of proliferating cell nuclear antigen (PCNA) by non-proliferating hepatocytes adjacent to metastatic tumours and in inflammatory conditions. F Pathol 1993;171:115-22.

29 Golick ML, Rice M. Optimum staining of PCNA in paraf fin sections is dependent on fixation, drying, and intensification. F Histotechnol 1992;15:39-41.

30 Kraaz W, Risberg B, Hussein A. Multiblock: An aid in diagnostic immunohistochemistry. F Clin Pathol 1988; 41:1337.

31 Brown DC, Gatter KC. Comparison of proliferation rate assessed using "multiblock" and conventional tissue blocks of lung carcinoma. $\mathcal{F}$ Clin Pathol 1992;45:579-82.
32 Cattoretti G, Pileri S, Parravicini C, Becker MHG, Poggi S, Bifulco C, et al. Antigen unmasking on formalin-fixed, paraffin-embedded tissue sections. F Pathol 1993;171: 83-98

33 Maddox PH, Jenkins D. 3 Aminopropyltriethoxysilane (APES): a new advance in section adhesion. $7 \mathrm{Cli}$ Pathol 1987;40:1256-7.

34 Thorpe SM, Rose C, Rasmussen BB, Mouridsen HT, Bayer T, Keilding N. Prognostic value of steroid hormone receptors: Multivariate analysis of systematically untreated patients with node negative primary breast cancer. Cancer Res 1987;47:6126-33.

35 Snead DRJ, Bell JA, Dixon AR, Nicholson RI, Elston CW, Blamey RW, et al. Methodology of immunohistological detection of oestrogen receptor in human breast carcidetection of oestrogen receptor in human breast carci-
noma in formalin-fixed, paraffin-embedded tissue: comparison with frozen section morphology. Histopathology 1993;23:233-8

36 McCormick D, Hall PA. The complexities of proliferating cell nuclear antigen. Histopathology 1992;21:581-4.

37 Bravo R, Macdonald Bravo H. Existence of two populations of cyclin/proliferating cell nuclear antigen durin the cell cycle: Association with DNA replication sites. Cell Biol 1987;105:1549-54.

38 Lynch DAF, Clarke AMT, Jackson P, Axon ATR, Dixon $M F$, Quirke $P$. Comparison of labelling by bromodeoxyuridine, MIB-1, and proliferating cell nuclear antideoxyuridine, Mr-1, and proliferating cell nuclear antigen in gastric m.

39 Norton AJ. Microwave oven heating for antigen unmasking in routinely processed tissue sections. I Patho 1993;171:79-80.

40 Charalambous C, Singh N, Isaacson PG. Immunohistochemical analysis of Hodgkin's disease using microwave heating. F Clin Pathol 1993;46:1085-8.

41 Gerdes J, Becker MHG, Key G. Immunohistochemica detection of tumour growth fraction (Ki-67 antigen) in formalin-fixed and routinely processed tissues. $₹$ Patho 1992;168:85-7.

42 Manakata S, Hendricks JB. Effect of fixation time and microwave oven heating time on retrieval of the Ki67 antigen from paraffin embedded tissue. $\mathcal{f}$ Histochem Cytochem 1993;41:1241-6. 